\title{
BMJ Open Randomised sham-controlled double- blind trial evaluating remote ischaemic preconditioning in solid organ transplantation: a study protocol for the RIPTRANS trial
}

\author{
Aki Uutela (1) , ${ }^{1}$ Ilkka Helanterä, ${ }^{1}$ Karl Lemström, ${ }^{2}$ Arie Passov, ${ }^{3}$ Simo Syrjälä, ${ }^{2}$ \\ Fredrik Åberg, ${ }^{1}$ Heikki Mäkisalo, ${ }^{1}$ Arno Nordin, ${ }^{1}$ Marko Lempinen, ${ }^{1}$ Ville Sallinen, ${ }^{1}$ \\ On behalf of the RIPTRANS Study Group collaborators
}

To cite: Uutela A, Helanterä I, Lemström K, et al. Randomised sham-controlled doubleblind trial evaluating remote ischaemic preconditioning in solid organ transplantation: a study protocol for the RIPTRANS trial. BMJ Open 2020;10:e038340. doi:10.1136/ bmjopen-2020-038340

- Prepublication history and additional material for this paper is available online. To view these files, please visit the journal online (http://dx.doi.org/10. 1136/bmjopen-2020-038340).

Received 09 March 2020 Revised 13 October 2020 Accepted 20 0ctober 2020

Check for updates

(C) Author(s) (or their employer(s)) 2020. Re-use permitted under CC BY-NC. No commercial re-use. See rights and permissions. Published by BMJ.

For numbered affiliations see end of article.

Correspondence to

Dr Ville Sallinen;

ville.sallinen@helsinki.fi

\section{ABSTRACT}

Introduction Remote ischaemic preconditioning (RIPC) using a non-invasive pneumatic tourniquet is a potential method for reducing ischaemia-reperfusion injury. RIPC has been extensively studied in animal models and cardiac surgery, but scarcely in solid organ transplantation. RIPC could be an inexpensive and simple method to improve function of transplanted organs. Accordingly, we aim to study whether RIPC performed in brain-dead organ donors improves function and longevity of transplanted organs.

Methods and analyses RIPTRANS is a multicentre, sham-controlled, parallel group, randomised superiority trial comparing RIPC intervention versus sham-intervention in brain-dead organ donors scheduled to donate at least one kidney. Recipients of the organs (kidney, liver, pancreas, heart, lungs) from a randomised donor will be included provided that they give written informed consent. The RIPC intervention is performed by inflating a thigh tourniquet to $300 \mathrm{~mm} \mathrm{Hg} 4$ times for $5 \mathrm{~min}$. The intervention is done two times: first right after the declaration of brain death and second immediately before transferring the donor to the operating theatre. The sham group receives the tourniquet, but it is not inflated. The primary endpoint is delayed graft function (DGF) in kidney allografts. Secondary endpoints include short-term functional outcomes of transplanted organs, rejections and graft survival in various time points up to 20 years. We aim to show that RIPC reduces the incidence of DGF from $25 \%$ to $15 \%$. According to this, the sample size is set to 500 kidney transplant recipients.

Ethics and dissemination This study has been approved by Helsinki University Hospital Ethics Committee and Helsinki University Hospital's Institutional Review Board. The study protocol was be presented at the European Society of Organ Transplantation congress in Copenhagen 14-15 September 2019. The study results will be submitted to an international peer-reviewed scientific journal for publication.

Trial registration number NCT03855722.

\section{Strengths and limitations of this study}

- The study method, a multicentre, double-blinded, sham-controlled, randomised superiority trial, is the best available method to investigate the effects of remote ischaemic preconditioning (RIPC) performed in the donor on the function and longevity of transplanted organs in the recipient.

- RIPC is an extremely simple, reproducible and inexpensive method.

- The sample size, 500 kidney transplant recipients, is large enough to provide confidence in the estimates of outcomes.

- Primary outcome, delayed graft function of kidney allograft, is clinically highly relevant, easy to measure and objective.

- As the sample size is calculated for kidney transplantation, outcomes of other organ recipients might be underpowered.

\section{INTRODUCTION}

Solid organ transplantation is an established standard of care for end-stage dysfunction of different organs, but the availability of the treatment is greatly limited globally by the shortage of organ donors. On the other hand, the lifetime of a transplanted organ is often limited and there is a number of patients waiting for a second or subsequent transplant. ${ }^{1-6} \mathrm{~A}$ transplanted organ is exposed to ischaemia-reperfusion injury during the transplantation process. $^{7}$ Alleviating this injury could improve the function and lifetime of transplanted organs.

Remote ischaemic preconditioning (RIPC) is an old concept where remotely produced ischaemia induces protective changes in distant organs or tissues and renders them less susceptible for future ischaemia via 
hormonal, metabolic and neuronal mechanisms. ${ }^{8}$ As an intervention, RIPC is easy and cheap to performan inflatable tourniquet is used to occlude upper or lower limb. RIPC has been extensively studied in animal models, ${ }^{9-11}$ and in human clinical trials of cardiac surgery. The largest of these clinical trials-RIPHeart, ${ }^{12}$ ERICCA $^{13}$ and CONDI-2/ERIC-PPCI ${ }^{14}$-have not been successful to show benefit from RIPC, but this might be due to the fact that the patients suffering from chronic myocardial ischaemia already have maximal compensatory mechanisms in use. This could also partially explain the results of RenalRIP trial, in which RIPC reduced acute kidney injury associated with cardiac surgery without affecting cardiac parameters. ${ }^{15}$

One of the postulated reasons for negative results in RIPHeart and ERICCA trials is the use of propofol instead of the volatile anaesthetics, even though this has not been fully verified. ${ }^{16}$ In the donation after brain death (DBD) transplantation setting, when the RIPC intervention is done to a brain dead donor, propofol is not used and should not prevent the effect of RIPC. Propofol may be used in the recipient surgery, but there is at least preliminary small animal data, that this may not prevent effectiveness of RIPC. ${ }^{17}$

Organ transplantation is a lucrative field to study RIPC, as the donor organs are healthy, and do not suffer from chronic ischaemia, but face invariable acute ischaemia of various durations. RIPC has been studied little in clinical transplantation and results have been controversial. An RIPC intervention done to heart transplant recipients together with post conditioning $20 \mathrm{~min}$ after aortic declamping reduced cTnI levels at 6 hours after transplantation. ${ }^{18}$ In a recent trial RIPC done to living liver donors reduced postoperative aspartate aminotransferase levels in liver recipients, ${ }^{19}$ whereas a pilot study of the RIPCOLT trial with RIPC done on liver transplant recipients demonstrated no short-term benefits. ${ }^{20}$

Direct ischaemic preconditioning with clamping of liver hilum in DBD was not was not beneficial and could even induce excessive ischaemic damage. ${ }^{21} \mathrm{~A}$ retrospective post hoc analysis of two such trials showed that liver ischaemic conditioning had no RIPC effect for kidneys. ${ }^{22}$ The liver-RIPC may provide an insufficient stimulus for the kidneys and the authors speculated that limb ischaemia could be a better method for RIPC. In a study of 29 kidney transplant patients RIPC done on DBD donors resulted in lower creatinine levels at 15 and 30 days after transplantation, but the change in glomerular filtration rate did not reach statistical significance. ${ }^{23}$ As far as we know, no larger randomised controlled trial with limbRIPC on DBD donors have been published.

A small study with 20 living donor kidney recipients per group found no difference in kidney function whether RIPC was done on donor or recipient. ${ }^{24}$ A larger trial of 170 living kidney donor-recipient pairs with RIPC done on donors reported lower postoperative creatinine values on donors after RIPC but no long-term benefits for donors or recipients. ${ }^{25}$
The largest kidney transplant RIPC trial to date, the REPAIR trial, showed that a RIPC performed in both donor and recipient immediately before a living-donor kidney surgery improved the estimated glomerular filtration for the whole follow-up period of 5 years. ${ }^{26}{ }^{27}$ The kidney allografts from living donors are subjected to very short ischaemia (in Finland this is typically less than 2 hours) and even greater benefits could be obtained if RIPC is performed in deceased donors, where ischaemia times are much longer (median 15 hours for kidney allografts in Finland, even longer in other countries). RIPC intervention performed to the recipients of deceased donor kidneys during the transplantation surgery did not improve kidney function in CONTEXT trial. ${ }^{26}$ This study can be criticised for performing RIPC in the recipients instead of donors, because the ischaemic injury has already taken place before RIPC.

The aim of this study is to show that RIPC performed in brain-dead donors (DBD) can be used to improve function and longevity of transplanted organs.

\section{METHODS AND ANALYSIS \\ Study design}

The RIPTRANS trial is a multicentre, double-blinded, parallel group, individual donor randomised superiority trial comparing RIPC with a sham-procedure performed in brain-dead donors. There is only one transplantation centre (Helsinki University Hospital) in Finland that covers the whole country and procurement team travels to all donor hospitals in Finland. This protocol was drafted in accordance with the Standard Protocol Items: Recommendations for Interventional Trials statement. ${ }^{27}$ Amendments made to the protocol can be found in supplemental (online supplemental file 1). This trial is registered in ClinicalTrials.gov, the first registration date was 27 February 2019.

\section{Participants}

RIPC or sham procedure will be performed on a braindead donor fulfilling inclusion and exclusion criteria. All brain-dead donors in participating hospitals scheduled for at least one kidney procurement will be included. Donors with significant haemodynamic instability (assessed by the intensive care physician responsible for the treatment of the donor) and under the age of 18 years will be excluded. Donors (or potential recipients of organ from this donor), who are participating in a trial with conflicting interventions or outcomes, will also be excluded. Although the donors are randomised and the intervention is carried out in donors, the recipients are the actual participants of this trial. All patients receiving a kidney, liver, combined pancreas-kidney, heart or lungs from a donor randomised in the trial will be included in the trial provided that they give a written informed consent (see online supplemental file 2) to participate in the trial and are at least 18 years old. The informed consent will be presented to the patient by a study nurse 
or physician. As based on the previous studies, RIPC is supposedly not harmful for the donor, and the lack of consent from any of the transplant recipients does not exclude the donor from the study, nor the possible inclusion of the other recipients. There are no other exclusion criteria for recipients who receive above-mentioned organs from a randomised donor.

\section{Randomisation and masking}

Eligible donors will be randomly allocated in a 1:1 ratio to either RIPC or sham-procedure group. The randomisation sequence was generated using a web-based commercial service (Sealed Envelope) with randomly variable block size $(4,6$ or 8$)$ and stratified according to donor age (under/over 60 years of age), planned organ to be procured (kidneys only/abdominal organs only/both thoracic and abdominal organs) and donor cardiopulmonary resuscitation (yes/no). The randomisation and allocation to either RIPC or sham-intervention is done by a transplant coordinator, who is not blinded to the allocated treatment, using the same web-based service. Once the donor is allocated, the transplant coordinator sends electronically or via fax written instructions on how to perform the allocated treatment to the intensive care team responsible for the treatment of the donor, who also are not blinded to the treatment. This intensive care team will collect data regarding the actual timing of the allocated procedure and whether this caused any noticeable changes in the donor hemodynamics. All researchers and all other treating personnel are blinded, such as procuring surgeons, transplant surgeons, treating physicians, data collectors and data analysts as well as recipients. After the trial recruitment has been closed and data collected, the allocated group will be named as A and B before the data are analysed. Once the data analyses for primary and secondary outcomes are completed, the full blinding will be removed. No emergency unblinding is planned, but incidents of possible breaches in blinding will be recorded.

\section{Procedures}

RIPC will be performed as follows: donor's thigh will be occluded 4 times for 5 min using tourniquet inflated to $300 \mathrm{~mm} \mathrm{Hg}$ each followed by $5 \mathrm{~min}$ of deflation. The intervention will be performed two times (once in both thighs). Once as soon as possible after brain death is determined, and once right before transferring the donor to the operation room for procurement. Sham-intervention will be performed by putting the inflatable tourniquet in place similarly, but not inflating it. Apart from the RIPC or sham-intervention, the treatment of donors will be according to normal routine. Study blood samples will be acquired from donors before (selected centres) and after the intervention.

\section{Outcomes}

The primary outcome measure is DGF of kidney allografts, which is defined as the need for dialysis within the first week after transplantation. Secondary outcome measures are different for different organs (box 1). Outcomes are assessed during the primary hospital stay, and thereafter at the routine follow-up visits. Helsinki University Hospital has a legal requirement to maintain a registry of all patients receiving a transplant in Finland, and data regarding visits in other hospitals are submitted to Helsinki University Hospital for registry purposes. Secondary outcomes are assessed directly from the registry, from the data provided by other hospitals, or at routine follow-up visits at Helsinki University Hospital. Survival status is automatically updated to the registry from the National Population Centre, which is an exact, complete, and up-to-date source for causes of death in Finland. Prespecified subgroup analyses are planned for characteristics that may potentially affect the results (table 1). Further exploratory outcome measures will be done according to online supplemental file 3 . In the informed consent, the patients are also asked to give their permission for using the excess study blood, urine and tissue samples in possible ancillary analysis.

\section{Statistical analyses}

The incidence of DGF in kidney allografts after transplantation from a brain-dead donor in Finland is approximately 25\% (Finnish Transplantation Registry). We aim to show that RIPC reduces the incidence of DGF to $15 \%$. With a $5 \%$ significance level and $80 \%$ power, 496 kidney transplantations are required to show this difference. Sample size is not adjusted for cross-over or lossof-follow up because the risk of these are considered to be minimal. Usually two kidneys per donor are transplanted. Because a portion of procured kidneys will be transferred to another Nordic country according to ScandiaTransplant rules, are untransplantable, transplanted in a combined organ transplantation, or transplanted to a recipient below 18 years old, we assume $90 \%$ of donors will lead to two kidney transplantations and $10 \%$ will lead to one kidney transplantation within the study. We set the final sample size to 500 kidney transplantations, for which approximately 260 donors are required to be randomised.

The primary outcome measure and the secondary outcomes for kidney transplantation will be analysed using generalised linear mixed models taking into account that kidneys from a single donor will usually be transplanted to two recipients included in the study. Survival analysis for kidney allografts and transplant recipients are done using Kaplan-Meier survival diagrams and the effect size is estimated using Cox proportional hazards regression model similarly taking into account single donor providing kidneys to two recipients.

The categorical outcome variables for liver, pancreas, heart and lungs are analysed with $\chi^{2}$ test (or Fischer's exact test, if $\mathrm{n}$ is under 5 in any of the subcategories). The continuous outcome variables for these organs are analysed using independent t-test or Mann-Whitney $\mathrm{U}$ test depending on whether the outcome has normal distribution or not. The effect size for categorical variables is 


\section{Box 1 Secondary outcome measures}

\section{Kidney allografts}

- Estimated glomerular filtration rate at 3 months, 1 year, 2 years, 5 years, 10 years and 20 years.

- Biopsy-proven acute rejection (BPAR) within 1 year.

- Graft survival at 3 months, 1 year, 2 years, 5 years, 10 years and 20 years: time from transplantation to death, retransplantation or permanent dialysis.

- Death-censored graft survival at 3 months, 1 year, 2 years, 5 years, 10 years and 20 years: time from transplantation to retransplantation or permanent dialysis, death-censored.

\section{Pancreatic allografts}

Glycosylated haemoglobin at 3 months, 1 year, 2 years, 5 years, 10 years and 20 years.

- Acute rejection in pancreatic allograft, either biopsy-proven (allograft pancreas or duodenal biopsy) acute rejection or clinically treated suspected acute rejection within 1 year.

- Pancreatic allograft survival at 3 months, 1 year, 2 years, 5 years, 10 years and 20 years: time from transplantation to death, retransplantation, explantation or daily insulin dependence.

- Death-censored pancreatic allograft survival at 3 months, 1 year, 2 years, 5 years, 10 years and 20 years: time from transplantation to death, retransplantation, explantation or daily insulin dependence, death-censored.

Liver allografts

- MEAF-score at third postoperative day (POD): Model for Early Allograft Function Scoring. MEAF=score ALTmax:3POD+score INRmax: 3POD+score bilirubin3POD, score range 0-10, higher score indicates worse outcome. ${ }^{30}$

- Postoperative biliary complications within 1 year: amount and type of postoperative biliary complications: stricture at anastomosis, bile leak or ischaemic type biliary lesions (ITBL) requiring intervention (endoscopic retrograde cholangiography, percutaneous transhepatic catheter, operation) or prolonged drainage within 1 year.

- Post-transplantation kidney injury (acute kidney injury) within 1 week, at 3 months, 1 year: according to Acute Dialysis Quality Initiative (ADQI) 2010 criteria. $^{31}$

BPAR within 1 year.

- Graft survival at 1 year, 2 years, 5 years, 10 years and 20 years: time from transplantation to death, retransplantation or explantation.

\section{Heart allografts}

- Ischaemia-reperfusion injury determined by peripheral blood Tnl levels at 6 hours hours after transplantation.

- Peripheral blood proBNP measurement at 1 week after transplantation.

- Primary graft dysfunction according to the International Society for Heart \& Lung Transplantation (ISHLT) definition ${ }^{32}$ within 24 hours after transplantation.

- Biopsy-proven or clinically treated acute rejection within 1 year after transplantation.

- Vasculopathy-free survival according to ISHLT definition ${ }^{33}$ at 1 year, 2 years, 5 years, 10 years and 20 years.

- Graft survival at 1 year, 2 years, 5 years, 10 years and 20 years, time from transplantation to death, retransplantation or explantation.

Lung allografts

- Primary graft dysfunction according to ISHLT definition ${ }^{34}$ within 72 hours after transplantation.

- Biopsy proven or clinically treated acute rejection within 1 year.

- Chronic lung allograft dysfunction free survival according to ISHLT/ American Thoracic Society (ATS)/European Respiratory Society

Continued
Box 1 Continued

(ERS) 2014 guideline $^{35}$ at 1 year, 2 years, 5 years, 10 years and 20 years, time from transplantation to death or retransplantation.

- Graft survival at 1 year, 2 years, 5 years, 10 years and 20 years: time from transplantation to death, retransplantation or explantation.

calculated with OR and 95\% CI. For continuous variables the effect size is calculated with difference in means with $95 \%$ CI for variables with normal distribution. If a continuous variable can be converted for normal distribution with a logarithmic transformation, will the effect size be reported using the ratio of geometrical means with $95 \%$ CI. Other continuous variables will be calculated using Mann-Whitney $\mathrm{U}$ test and the effect size will be reported using $\mathrm{r}=\mathrm{Z} / \sqrt{ } \mathrm{N}$ without $95 \%$ CI. Survival analysis for these organs will be described using Kaplan-Meier survival diagrams and log-rank test and effect size estimated using Cox proportional hazards regression model.

Subgroup analysis will be made using generalised linear mixed models as univariate analysis by adjusting models by subgroup. A multivariate analysis of subgroups can be done with aforementioned generalised linear model and by selecting the significant subgroups $(p<0.05)$ from univariate analysis as covariates. Subgroup analysis for survival variables will be described with Kaplan-Meier, which will be stratified by subgroup and effect size will be estimated using Cox proportional hazards regression model by adjusting it with the subgroup.

In case that because of missing values more than 5\% of patients would be left out from sensitivity analyses, multiple imputations may be used to conduct sensitivity analyses. Otherwise, the missing data will not be adjusted separately, but these cases will either be left out from the analyses or censored at the last point of follow-up.

\section{Data security}

All patient data included in the study is confidential and will be concealed on a computer behind an Advanced Encryption Standard (AES) 256-bit encryption. Any data stored in a paper from will be held in the study hospitals

\begin{tabular}{|c|c|}
\hline Subgroup analyses & Subgroups \\
\hline $\begin{array}{l}\text { Donor cardiopulmonary } \\
\text { resuscitation }\end{array}$ & Yes/no \\
\hline Donor age (years) & Under 60/over 60 \\
\hline Donor sex & Male/female \\
\hline $\begin{array}{l}\text { Organ cold ischaemia time } \\
\text { (hours, organ specific) }\end{array}$ & Below/above median \\
\hline Uncompleted study intervention & Yes/no \\
\hline $\begin{array}{l}\text { Liver transplantation for acute } \\
\text { liver failure }\end{array}$ & Yes/no \\
\hline
\end{tabular}


in locked offices. Only the study personnel will have the access to the trial dataset.

\section{Schedule and interim safety analyses}

The study was conceptualised in June 2017. The study plan was approved by the Helsinki University Hospital's Ethics Committee 9 May 2018. Helsinki University Hospital's Institutional Review Board gave permission to conduct the study 14 August 2018. The study started recruiting in 13 May 2019 in four out of five university hospitals in Finland. The Ethics Committee required a safety analysis after 16 donors had been randomised. The donors and recipients of kidneys from these 16 donors were analysed without unmasking the allocated group. All 16 donors randomised successfully underwent procurement. No adverse events were noted in the recipients. After this safety analysis, the study will be disseminated to non-university donor hospitals. Second interim analysis will be done when half of the target sample size is reached (250 kidney transplantations). In Finland, approximately 230 DBD kidney transplants are being performed annually. We estimate that data for primary outcome would be available in 4 years.

\section{Role of the funding sources and sponsors}

The funders or sponsors have had and will have no role in study design, data collection, data analysis, data interpretation, or writing the report, or any other aspect of the work, except for funding.

\section{Patient and public involvement}

Patient organisations were not involved in the study design.

\section{Ethics and dissemination}

\section{Study ethics}

This study has been approved by Helsinki University Hospital Ethics Committee and Helsinki University Hospital's Institutional Review Board.

The intervention is performed on a donor, who has been determined brain dead and has given permission to act as a donor according to Finnish legislation, and is determined suitable and scheduled for kidney procurement. The Ethics Committee has approved that donors (or next of kin) do not need to consent to RIPC or sham-procedure because it is a non-invasive procedure, the donor is brain dead and scheduled for procurement already.

The recipients of organs from randomised donors will be recruited in the study and will be required to give written informed consent to participate. The recipient cannot influence whether the donor has been randomised or received the allocated treatment. The recipient has the right to decline participation in the trial, but can still choose to receive the planned allograft. In these cases, the recipient's data are not used in the study analyses. The recipient has also right to decline the offered organ. The recipient does not have the right to know the allocated treatment the donor has received before the study has been completed, data analysed and blinding unmasked. The donors or recipients do not receive any compensation for their participation in the trial. The recipients have the right to discontinue the trial or withdraw their consent at any point. In these cases, the collected data will be used in the analyses up to the point of discontinuation.

A few additional blood samples (and a urine sample from the kidney recipients) will be taken from the kidney, heart and lung recipients for the study purposes during and shortly after the transplantation, but otherwise the recipients only give their consent to the study group to observe and collect medical information. These samples are stored maximally for 5 years after the completion of the study recruitment. The patient informed consent forms are in Finnish and Swedish and will be provided by request made to the study group.

\section{Harms}

Earlier studies on RIPC have not indicated any harm (7-16). On the contrary, many earlier studies suggest that RIPC may be beneficial for the function and longevity of the allografts. Before wider adoption of the RIPC in transplantation, its safety and benefits need to be addressed in a randomised controlled trial such as RIPTRANS. Any possible harmful effects of the intervention will be reported together with the study results. The Finnish patient insurance covers the organ recipients participating in the study.

\section{Monitoring}

Helsinki University Hospital Ethics Board monitored the results of the first interim analysis. Initially the Ethics board did not necessitate a separate Data Monitoring Committee (DMC). To provide external validity for the study, a DMC contract was made with Clinical Research Institute HUCH (HYKS Instituutti) in March 2020. The site monitoring will be performed every 3 months including review of the Investigator's Trial File, facilities, the equipment at the site, compliance to study protocol and study specific procedures, source document quality and the intervention implementation documentation for all donors. All the study patients will be monitored for: existence, informed consent process and documentation of the trial outcome measures. A complete review will be conducted for $10 \%$ of the subjects. A close-out visit shall be done after all the data have been collected and the treatment of all the subjects has been completed. This monitoring plan and agreement is made in collaboration with the guideline for coordinated Good Clinical Practive (GCP)-monitoring of clinical trials in the Nordic countries (V.5/24 October 2017).

\section{Dissemination}

The study protocol was be presented at the European Society of Organ Transplantation congress in Copenhagen 14-15 September 2019 and will possibly be presented in other scientific conferences. The study results will be submitted to an international peer-reviewed scientific 
journal for publication and possibly discussed at scientific meetings. The study is also being made public via social media platforms (Twitter). The International Committee of Medical Journal Editors recommendations (www. ICMJE.org) are applied when considering the authorship of any publications from this trial.

\section{DISCUSSION}

We formed our study protocol based on earlier RIPC studies. The RIPC intervention on transplant recipients has already been thoroughly studied by Krogstrup $e t a l^{26}$ and we saw more potential in performing the intervention prior to organ procurement, in deceased donors. Our RIPC tourniquet protocol is similar to several earlier trials. ${ }^{12-14} 26$ Our intervention is performed on thigh (instead of upper extremity), which has a larger mass and could thus produce larger effect, and donor arms are usually used for cannulas which could cause interference with RIPC. A constant tourniquet pressure of $300 \mathrm{~mm} \mathrm{Hg}$ was selected because it ensures that the circulation to the lower extremity is ceased and ischaemia introduced. Also, a static pressure protocol instead of changing the pressure according to systolic blood pressure is simpler and easier to reproduce.

The aim of our study is to be able to show a decrease in DGF rate from $25 \%$ to $15 \%$. The largest kidney transplant RIPC trial thus far, the REPAIR trial, recruited 406 kidney recipients and the reported a positive long-term outcome for kidney function, but not in DGF. ${ }^{28} 29$ A DGF difference in living donor setting is hard to show because of very low incidence of DGF compared with deceased donation. A DGF reduction of 10 percentage points was chosen because there were several reasons assume that the effect of RIPC would be higher in our study. First, the intervention is performed on thigh with larger mass compared with arm as noted above. Second, intervention is performed on DBD donors, in which both warm and cold ischaemia times are longer than in living donors, where the earlier trials have mostly been conducted. Further, we think that the effect of RIPC could be larger because of the systemic inflammatory cascade in DBD donors, which RIPC might be able to alleviate. All in all, these are hypotheses, which are now being tested in a novel double-blind randomised controlled trial. An interim analysis will be made when half of the sample size is recruited (250 kidney transplant recipients) and at that point, we will acquire a more precise estimate of the effect.

\section{Author affiliations}

${ }^{1}$ Department of Transplantation and Liver Surgery, Helsinki University Hospital and University of Helsinki, Helsinki, Finland

${ }^{2}$ Department of Cardiothoracic Surgery, Helsinki University Hospital and University of Helsinki, Helsinki, Finland

${ }^{3}$ Department of Perioperative, Intensive Care and Pain Medicine, Helsinki University Hospital and University of Helsinki, Helsinki, Finland

Twitter Aki Uutela @Aki_Uutela
Acknowledgements The authors wish to thank all the collaborators of this study, and in particular Helsinki University Hospital transplant coordinators Siv Ansa, Carola Schauman, Leena Toivonen, Eero Hartikka and Heikki Norio, the personnel of the participating intensive care units andthe personnel of the Meilahti Hospital operating theatre and the transplantation wards.

Collaborators Minna Bäcklund (Department of Perioperative, Intensive Care and Pain Medicine, Helsinki University Central Hospital and University of Helsinki); Markus Skrifvars (Department of Emergency Care and Services, Helsinki University Central Hospital andUniversity of Helsinki); Teemu Luostarinen (Department of Perioperative, Intensive Care and Pain Medicine, Helsinki University Central Hospital and University of Helsinki); Janne Reitala (Department of Perioperative, Intensive Care and Pain Medicine, Helsinki University Central Hospital and University of Helsinki); Maarit Lång (Department of Intensive Care Medicine, Kuopio University Hospital, Kuopio, Finland); Ilona Leppänen (Department of Intensive Care, Tampere University Hospital, Tampere, Finland); Jaakko Långsjö (Department of Intensive Care, Tampere University Hospital, Tampere, Finland); Juha Grönlund (Department of Intensive Care, Turku University Hospital, Turku, Finland); Pekka Loisa (Department of Intensive Care, Päijät-Häme Central Hospital, Lahti, Finland); Anni Pulkkinen (Department of Intensive Care, Central Finland Central Hospital, Jyväskylä, Finland); Björn Jäschke (Department of Intensive Care, Satakunta Central Hospital, Pori, Finland).

Contributors Concept: VS, ML. Initial draft of protocol: VS, AU, IH, KL. Critical revision of the protocol: VS, AU, IH, KL, AP, SS, FÅ, HM, AN, ML. Implementation: VS, AU, KL, SS, AP. Data collection: AU, SS. Donor recruitment (Study Group Collaborators): MB, MS, TL, JR, MaL, IL, JL, JG, PL, AP, BJ.

Funding This work was supported by the Academy of Finland, Finska Läkaresällskapet and Helsinki University Hospital's research funds.

Sponsorship statement The study sponsor is Helsinki University Hospital. Competing interests None declared.

Patient consent for publication Not required.

Provenance and peer review Not commissioned; externally peer reviewed.

Supplemental material This content has been supplied by the author(s). It has not been vetted by BMJ Publishing Group Limited (BMJ) and may not have been peer-reviewed. Any opinions or recommendations discussed are solely those of the author(s) and are not endorsed by BMJ. BMJ disclaims all liability and responsibility arising from any reliance placed on the content. Where the content includes any translated material, BMJ does not warrant the accuracy and reliability of the translations (including but not limited to local regulations, clinical guidelines, terminology, drug names and drug dosages), and is not responsible for any error and/or omissions arising from translation and adaptation or otherwise.

Open access This is an open access article distributed in accordance with the Creative Commons Attribution Non Commercial (CC BY-NC 4.0) license, which permits others to distribute, remix, adapt, build upon this work non-commercially, and license their derivative works on different terms, provided the original work is properly cited, appropriate credit is given, any changes made indicated, and the use is non-commercial. See: http://creativecommons.org/licenses/by-nc/4.0/.

ORCID iD

Aki Uutela http://orcid.org/0000-0003-1324-5894

\section{REFERENCES}

1 Hart A, Smith JM, Skeans MA, et al. OPTN/SRTR 2017 annual data report: kidney. Am J Transplant 2019;19:19-123.

2 Kandaswamy R, Stock PG, Gustafson SK, et al. OPTN/SRTR 2017 annual data report: pancreas. Am J Transplant 2019;19:124-83.

3 Kim WR, Lake JR, Smith JM, et al. OPTN/SRTR 2017 annual data report: liver. Am J Transplant 2019;19:184-283.

4 Colvin M, Smith JM, Hadley N, et al. OPTN/SRTR 2017 annual data report: heart. Am J Transplant 2019;19:323-403.

5 Valapour M, Lehr CJ, Skeans MA, et al. OPTN/SRTR 2017 annual data report: lung. Am J Transplant 2019;19:404-84.

6 Israni AK, Zaun D, Rosendale JD, et al. OPTN/SRTR 2017 annual data report: deceased organ donation. Am J Transplant 2019;19:485-516.

7 Aydin Z, van Zonneveld AJ, de Fijter JW, et al. New horizons in prevention and treatment of ischaemic injury to kidney transplants. Nephrology Dialysis Transplantation 2007;22:342-6. 
8 Kanoria S, Jalan R, Seifalian AM, et al. Protocols and mechanisms for remote ischemic preconditioning: a novel method for reducing ischemia reperfusion injury. Transplantation 2007;84:445-58.

9 Murry CE, Jennings RB, Reimer KA. Preconditioning with ischemia: a delay of lethal cell injury in ischemic myocardium. Circulation 1986;74:1124-36.

10 Przyklenk K, Bauer B, Ovize M, et al. Regional ischemic 'preconditioning' protects remote virgin myocardium from subsequent sustained coronary occlusion. Circulation 1993;87:893-9.

11 Kharbanda RK, Mortensen UM, White PA, et al. Transient limb ischemia induces remote ischemic preconditioning in vivo. Circulation 2002;106:2881-3.

12 Meybohm P, Bein B, Brosteanu O, et al. A multicenter trial of remote ischemic preconditioning for heart surgery. $N$ Engl $\mathrm{J}$ Med 2015;373:1397-407.

13 Hausenloy DJ, Candilio L, Evans R. Effect of remote ischaemic preconditioning on clinical outcomes in patients undergoing coronary artery bypass graft surgery (ERICCA study): a multicentre doubleblind randomised controlled clinical trial. efficacy and mechanism evaluation. Southampton (UK): NIHR Journals Library, 2016.

14 Hausenloy DJ, Kharbanda RK, Møller UK, et al. Effect of remote ischaemic conditioning on clinical outcomes in patients with acute myocardial infarction (CONDI-2/ERIC-PPCI): a single-blind randomised controlled trial. The Lancet 2019;394:1415-24.

15 Zarbock A, Schmidt C, Van Aken H, et al. Effect of remote ischemic preconditioning on kidney injury among high-risk patients undergoing cardiac surgery. JAMA 2015;313:2133-41.

16 Benstoem C, Goetzenich A, Autschbach R, et al. Volatile anesthetics versus propofol in the cardiac surgical setting of remote ischemic preconditioning: a secondary analysis of a Cochrane systematic review. Minerva Anestesiol 2018;84:1298-306.

17 Chen K, Yu J, Wang Q, et al. The timing of propofol administration affects the effectiveness of remote ischemic preconditioning induced cardioprotection in rats. J Cell Biochem 2020;121:4535-41.

18 Wang G, Zhang Y, Yang L, et al. Cardioprotective effect of remote ischemic preconditioning with postconditioning on donor hearts in patients undergoing heart transplantation: a single-center, doubleblind, randomized controlled trial. BMC Anesthesiol 2019;19:48.

19 Jung K-W, Kang J, Kwon H-M, et al. Effect of remote ischemic preconditioning conducted in living liver donors on postoperative liver function in donors and recipients following liver transplantation. Ann Surg 2020;271:646-53.

20 Robertson FP, Goswami R, Wright GP, et al. Remote ischaemic preconditioning in orthotopic liver transplantation (RIPCOLT trial): a pilot randomized controlled feasibility study. HPB 2017;19:757-67.

21 Koneru B, Shareef A, Dikdan G, et al. The ischemic preconditioning paradox in deceased donor liver Transplantation-Evidence from a prospective randomized single blind clinical trial. Am J Transplant 2007;7:2788-96.

22 Desai KK, Mora-Esteves C, Holland BK, et al. Does liver ischemic preconditioning in brain death donors induce kidney preconditioning? A retrospective analysis. Transplantation Journal 2014;97:337-43.

23 Zapata-Chavira H, Hernández-Guedea M, Jiménez-Pérez JC, et al. Modulation of remote ischemic preconditioning by proinflammatory cytokines in renal transplant recipients. J Invest Surg 2019;32:63-71.

24 Chen $\mathrm{Y}$, Zheng $\mathrm{H}$, Wang $\mathrm{X}$, et al. Remote ischemic preconditioning fails to improve early renal function of patients undergoing living-donor renal transplantation: a randomized controlled trial. Transplantation 2013;95:e4-6.

25 Bang J-Y, Kim S-G, Oh J, et al. Impact of remote ischemic preconditioning conducted in living kidney donors on renal function in donors and recipients following living donor kidney transplantation: a randomized clinical trial. J Clin Med 2019;8:713.

26 Krogstrup NV, Oltean M, Nieuwenhuijs-Moeke GJ, et al. Remote ischemic conditioning on recipients of deceased renal transplants does not improve early graft function: a multicenter randomized, controlled clinical trial. Am J Transplant 2017;17:1042-9.

27 Chan A-W, Tetzlaff JM, Altman DG, et al. Spirit 2013 statement: defining standard protocol items for clinical trials. Ann Intern Med 2013;158:200-7.

28 MacAllister R, Clayton T, Knight R. Remote preconditioning for protection against ischaemia-reperfusion in renal transplantation (repair): a multicentre, multinational, double-blind, factorial designed randomised controlled trial. efficacy and mechanism evaluation. Southampton (UK): NIHR Journals Library, 2015.

29 Veighey KV, Nicholas JM, Clayton T, et al. Early remote ischaemic preconditioning leads to sustained improvement in allograft function after live donor kidney transplantation: long-term outcomes in the renal protection against Ischaemia-reperfusion in transplantation (repair) randomised trial. Br J Anaesth 2019;123:584-91.

30 Jochmans I, Fieuws S, Monbaliu D, et al. "Model for early allograft function" outperforms "early allograft dysfunction" as a predictor of transplant survival. Transplantation 2017;101:e258-64.

31 Nadim MK, Genyk YS, Tokin C, et al. Impact of the etiology of acute kidney injury on outcomes following liver transplantation: acute tubular necrosis versus hepatorenal syndrome. Liver Transpl 2012;18:539-48.

32 Kobashigawa J, Zuckermann A, Macdonald P, et al. Report from a consensus conference on primary graft dysfunction after cardiac transplantation. J Heart Lung Transplant 2014;33:327-40.

33 Mehra MR, Crespo-Leiro MG, Dipchand A, et al. International Society for heart and lung transplantation working formulation of a standardized Nomenclature for cardiac allograft vasculopathy-2010. J Heart Lung Transplant 2010;29:717-27.

34 Snell GI, Yusen RD, Weill D, et al. Report of the ISHLT Working group on primary lung graft dysfunction, part I: definition and grading-A 2016 consensus group statement of the International Society for heart and lung transplantation. J Heart Lung Transplant 2017;36:1097-103

35 Meyer KC, Raghu G, Verleden GM, et al. An international ISHLT/ ATS/ERS clinical practice guideline: diagnosis and management of bronchiolitis obliterans syndrome. Eur Respir J 2014;44:1479-503. 